\title{
Optimal parameters for Gene Xpert MTB/RIF and LAM Strip determine Test for Diagnosis of Tuberculosis using urine from HIV patients at Moi Teaching and Referral Hospital, Kenya
}

\author{
Authors \\ Iddah. M. Ali ${ }^{1,2}$, Gideon A. Ng'wena ${ }^{3}$, Benard. O. Guyah ${ }^{1}$ Kennedy K.Mutai $^{4}$, \\ Benard. O. Abong'o 1
}

${ }^{1}$ Dept of Biomedical Science and Technology, Maseno University, P.O. Box 333-40105, Maseno, Kenya

${ }^{2}$ Dept of Medical Laboratory Science, Moi Teaching and Referral Hospital, P.O. Box 3-30100, Eldoret, Kenya

${ }^{3}$ Dept of Medical Physiology, School of Medicine, Maseno University, P.O. Box 333-40105, Maseno, Kenya

${ }^{4}$ Academic Model Providing Access to Healthcare (AMPATH), Eldoret, Kenya

Email: iddah.ali@gmail.com ${ }^{1,2}$,gngwena@hotmail.com ${ }^{3}$,guyah.bernard@yahoo.com ${ }^{1}$, kkmuttai@gmail.com $^{4}$ andmelfelis@gmail.com ${ }^{1}$

Corresponding Author

\section{Iddah Maulid Ali}

Dept of Biomedical Science and Technology, Maseno University, P.O. Box 333-40105, Maseno, Kenya

${ }^{2}$ Dept of Medical Laboratory Science, Moi Teaching and Referral Hospital, P.O. Box 3-30100, Eldoret,

Kenya

\section{ABSTRACT}

The application of Gene Xpert MTB/RIF® assay for testing non-sputum clinical samples in Western Kenya has not been reported. We are reporting on use of $\mathrm{Zn}, \mathrm{CD} 4$ count, Hb, Creatinine, Proteinuria, and Hematuria as optimal parameters for Gene Xpert MTB/RIF and LAM Strip determine Test for diagnosis of tuberculosis. Urine from HIV/TB co-infected adults $(n=158)$ with CD4+ count $<200$ cells $/ \mathrm{mm}^{3}$ was analyzed for mycobacteria tuberculosis DNA. Acid fast-staining, culture, clinical symptoms, laboratory and radiological findings were also assessed. Acid fast staining(55\%) were TB positive, however, Gene Xpert test revealed 17(11\%) of the patients were TB positive with $94 \%$ and $6 \%$ being MTB detected low and medium, respectively. Rifampicin resistance was not detected in $88 \%$ of the TB Gene Xpert positive patients with the remaining (12\%) being indeterminate. LAM test revealed that $28 \%$ of the patients were TB positive. Radiology revealed that $45 \%$ of the patients had infiltrates. Presence of protein or blood in urine was significantly associated with TB positivity based on LAM strip determine test (48\% vs 24\%; p-value=0.021). The weighted kappa coefficient was 0.48 (95\% CI 0.32-0.63; exact p-value <0.0001). The findings of this study show that urine Xpert MTB/RIF® can be used for diagnosis of TB using urine and taking Zn, CD4 count, Proteinuria, and Hematuria as optimal parameters.

Keywords: Urine, mycobacterium, Proteinuria, Hematuria, HIV Infection

\section{INTRODUCTION}

HIV and tuberculosis combined are cause of death in patients with HIV. In 2010, 2.8 million new cases of tuberculosis were reported in Africa, the majority in the sub-Saharan area; and $37 \%$ of tuberculosis episodes were diagnosed among HIV 
infected patients ${ }^{1}$. In 2011, some 430,000 people died of HIV- associated TB, most of them in developing countries, and 22.5 million people were estimated to be living with HIV in subSaharan Africa ${ }^{2}$. The risk of tuberculosis increases with HIV but also modifies the clinical presentation of $\mathrm{TB}^{3}$. HIV-infected patients experience more sputum smear-negative pulmonary tuberculosis (PTB) than HIVuninfected patients with extra pulmonary tuberculosis (EPTB) being, contributing to delayed tuberculosis diagnosis and high mortality ${ }^{4}$ Alteration in the clinical and radiographic presentation of PTB among HIVinfected has long been recognized ${ }^{5}$. Direct smears can be are often negative and cannot differentiate mycobacterium tuberculosis from non-tuberculous mycobacterium $^{6}$. Culture (standard) takes 2 to 8 weeks due to the slow growth rate of mycobacterium while liquid culture may take 7-10 days ${ }^{7}$.New innovative diagnostic methods for tuberculosis with increased sensitivity and speed of diagnosis in these patient groups are needed ${ }^{8}$, ${ }^{9}$.Urine can be cultured, tested by polymerase chain reaction (PCR) for mycobacterial transrenal DNA or for specific mycobacterial antigens (lipoarabinomannan (LAM)). Currently there is no adequate data on the diagnostic utility of urine in TB diagnosis ${ }^{10}$. Recently, the Gene Xpert MTB/RIF rapid molecular assay has been endorsed by the Scientific and Technical Advisory Board of the World Health Organization (WHO) as the most sensitive test for TB diagnosis in respiratory samples ${ }^{11,12}$. This study will determine optimal parameters for Gene Xpert MTB/RIF and LAM Strip determine test for diagnosis of Tuberculosis using HIV patients' urine.

\section{MATERIAL AND METHODS}

Study population: This study evaluated $158 \mathrm{HIV}$ patients attending Moi Teaching and Referral Hospital in Eldoret, Western Kenya. Urinalysis, complete blood count, biochemical analysis of urine samples was done on adults $(\geq 18)$ years with $\mathrm{CD} 4+<200$ cells $/ \mathrm{mm}^{3}$. Urine samples from patients with suspected TB were analyzed for mycobacteria tuberculosis DNA. Acid faststaining, culture, clinical symptoms, and radiological findings were also evaluated. Host factors (age, gender, patient category, site of TB, HIV status, CD+ count) was obtained from the hospital records.

Statistical Analysis: Statistical analysis was done using SAS version 9.3.Categorical variables were summarized as frequencies and percentages. Continuous variables were summarized as mean and standard deviation or median and the corresponding inter quartile range. Normality tests were conducted using Shapiro and Wilks normality test. The test for association between categorical variables were conducted using Pearson's Chi Square test while the test of association between continuous variables were conducted using a two sample t-test if they are normally distributed. For skewed variables, a two sample Wilcoxon rank sum test was used. Cohen's kappa statistic (k) test was used to measure the level of agreement between the Xpert $\mathrm{MTB} / \mathrm{RIF}$ and LAM determine strip test in detecting TB. Sensitivity, specificity, positive predictive value (PPV), and negative predictive value (NPV) with their 95\% confidence intervals (CI) were computed against the sputum microscopy method (gold standard). Logistic regression analysis was used to identify factors associated with urine MTB/RIF positivity.

Ethical considerations: Ethical approval (\# 0001084) was obtained from the Institution Review and Ethics Committee (IREC) of MTR.

\section{RESULTS}

A total of 158 HIV positive patients were used as study participants. The overall mean age in years was 39 (SD, 9.1). Females formed 54\% of the population. A third $(31 \%)$ of the patients had CD4 count $\leq 100$ cells $/ \mathrm{mm}^{3}$. WHO disease progressions of patients were either in their $3^{\text {rd }}$ or $4^{\text {th }}$ stage of HIV. The median hemoglobin level was 12.0 (IQR, 10.1-13.6) (Table 1). 


\section{JMSCR Vol.||03||Issue||08||Page 6997-7004||August}

Table 1: Demographic and clinical characteristics among HIV-infected patients in MTRH, 2011-2013

\begin{tabular}{ll}
\hline Characteristics & $\begin{array}{l}\mathrm{N}=158 \\
\mathrm{n},(\%)\end{array}$ \\
\hline Gender (Female) & $85(53.8)$ \\
Age (years), mean (std) & $38.7(9.1)$ \\
CD4 count, cell/mm $\mathrm{mm}^{3}$ & \\
$\quad \leq 100$ & $49(31.0)$ \\
$>100$ & $106(67.1)$ \\
$\quad$ Missing & $3(1.9)$ \\
WHO stage & \\
Stage I \& II & $25(15.8)$ \\
Stage III \& IV & $105(66.5)$ \\
$\quad$ Missing & $28(17.7)$ \\
Hemoglobin, median (IQR) & $12.0 \quad(10.1-13.6)$ \\
\hline
\end{tabular}

Clinical symptoms; weight loss, TB, Pneumonia, URTI and those with a combination of TB or
Pneumonia or Meningitis formed a cumulative of $73 \%(61 / 84)$ (Table 2).

Table 2: Clinical diagnoses among HIV-infected patients in MTRH, 2011-2013

\begin{tabular}{ll}
\hline Clinical symptom & $\mathrm{n}(\%)$ \\
\hline Adenitis, TB & $1(0.6)$ \\
Allergy & $1(0.6)$ \\
Anemia & $3(1.9)$ \\
Asymptomatic HIV & $3(1.9)$ \\
Bronchospasm & $1(0.6)$ \\
Conjunctivitis & $1(0.6)$ \\
Dehydration & $1(0.6)$ \\
Gastroenteritis & $2(1.3)$ \\
Herpes zoster & $3(1.9)$ \\
Hypertension & $2(1.3)$ \\
Meningitis & $1(0.6)$ \\
Migraine & $1(0.6)$
\end{tabular}


Milliary Tuberculosis

Pneumonia

Pneumonia, Tuberculosis

Pneumonia, Tuberculosis

Pericardial effusion

RTI, Cough

Tuberculosis

Tuberculosis meningitis

URTI

Weight loss

None
$5(3.2)$

$8(5.1)$

7 (4.4)

$1(0.6)$

$1(0.6)$

$10(6.3)$

$15(9.5)$

$1(0.6)$

$9(5.7)$

7 (4.4)

74 (46.8)
Median urine culture was 125 (IQR, 110-272). Majority (85\%) had nil results on urinalysis. Blood and Protein was reported in $65 \%$ and $26 \%$ respectively among those with non-nil result. A fifth $(28 \%)$ had positive acid fast and more than half $(55 \%)$ of these being TB positive. Gene Xpert test showed 17 (11\%) had TB positive of which $94 \%$ and $6 \%$ were MTB detected low medium, respectively. Rifampicin was not detected in $88 \%$ of the TB Gene Xpert positive patients. The remaining $(12 \%)$ being indeterminate. All patients were sensitive to drugs. Using LAM test, $28 \%$ of the patients tested positive for TB. Radiological findings were; $45 \%$ had infiltrates, $19 \%$ normal, $15 \%$ milliary $\mathrm{TB}$ and $11 \%$ opacities. Median creatinine was 62.3 (IQR 49.1-75.9)(Table 3).

Table 3: Microbiological and laboratory characteristics among HIV-infected patients in MTRH, 2011-2013

\begin{tabular}{|c|c|}
\hline Characteristics & $\mathrm{n},(\%)$ \\
\hline \multicolumn{2}{|l|}{ Urinalysis } \\
\hline+ BLOOD & $15(9.5)$ \\
\hline + BLOOD , + PROTEIN & $1(0.6)$ \\
\hline + BLOOD, ++ PROTEIN & $1(0.6)$ \\
\hline + PROTEIN & $6(3.8)$ \\
\hline NIL & $135(85.4)$ \\
\hline \multicolumn{2}{|l|}{ Acid Fast $(\mathrm{ZN})$} \\
\hline+ & $16(10.1)$ \\
\hline++ & $5(3.2)$ \\
\hline+++ & $8(5.1)$ \\
\hline NEG & $129(81.7)$ \\
\hline \multicolumn{2}{|l|}{ TB status (Gene Xpert) } \\
\hline MTB detected low & $16(10.1)$ \\
\hline MTB detected medium & $1(0.6)$ \\
\hline MTB not detected & $141(89.2)$ \\
\hline \multicolumn{2}{|l|}{ Rifampicin (RIF) resistant } \\
\hline RIF indeterminate & $2(11.8)$ \\
\hline RIF not detected & $15(88.2)$ \\
\hline \multicolumn{2}{|l|}{ Drug sensitivity } \\
\hline Streptomycin, Isoniazid, RIF, Ethambutol & $17(100.0)$ \\
\hline
\end{tabular}




\begin{tabular}{ll}
\hline None & $0(0.0)$ \\
LAM & \\
Negative & $114(72.2)$ \\
Positive & $44(27.8)$ \\
Urine culture & $125(110-272)$ \\
Radiological findings & \\
Cavitation & $2(1.3)$ \\
Adenitis & $3(1.9)$ \\
Infiltrates & $71(44.9)$ \\
Lung collapse & $1(0.6)$ \\
Milliary TB & $23(14.6)$ \\
Normal & $30(19.0)$ \\
Opacities & $18(11.4)$ \\
Pericarditis & $1(0.6)$ \\
Pleural effusion & $3(1.9)$ \\
Suggestive & $1(0.6)$ \\
Tb meningitis & $4(2.5)$ \\
Tb spine & $1(0.6)$ \\
Creatinine, median (IQR) & 62.3 \\
\hline
\end{tabular}

Presence of protein or blood in urine was on LAM strip determine test (48\% vs $24 \%$; psignificantly associated with TB positivity based value $=0.021)$ (Table 4).

Table 4: Association between Urinalysis result and TB results based on Gene Xpert result and LAM determine strip techniques

*significant at $\alpha=0.05$

TB Testing Technique

\begin{tabular}{|c|c|c|c|c|c|c|}
\hline \multirow[b]{3}{*}{ Urinalysis result } & \multicolumn{6}{|c|}{ TB Testing Technique } \\
\hline & \multicolumn{3}{|c|}{ Gene Xpert result } & \multicolumn{3}{|c|}{ LAM determine result } \\
\hline & $\begin{array}{l}\text { Negative } \\
\mathrm{n}(\%)\end{array}$ & $\begin{array}{l}\text { Positive } \\
\text { n }(\%)\end{array}$ & $\mathrm{P}$-value & $\begin{array}{l}\text { Negative } \\
\mathrm{n}(\%)\end{array}$ & $\begin{array}{l}\text { Positive } \\
\mathrm{n}(\%)\end{array}$ & $\mathrm{P}$-value \\
\hline \multicolumn{7}{|l|}{ Negative } \\
\hline \multirow[t]{2}{*}{ Positive } & $122(90)$ & $13(10)$ & 0.277 & $102(76)$ & $33(24)$ & $0.021 *$ \\
\hline & $19(83)$ & $4(17)$ & & $12(52)$ & $11(48)$ & \\
\hline
\end{tabular}

Weighted kappa coefficient was 0.48 (95\% CI amount of agreement between the Gene Xpert and $0.32-0.63$; exact p-value $<0.0001)$. Hence, the LAM was modest (Table 5). 
Table 5: Cohen's kappa statistic $(\mathrm{k})$ test for the agreement between the Gene Xpert MTB/RIF and LAM determine techniques in detecting TB

\begin{tabular}{|c|c|c|c|c|c|c|}
\hline \multirow[b]{2}{*}{$\begin{array}{l}\text { LAM } \\
\text { technique }\end{array}$} & \multirow[b]{2}{*}{ determine } & \multicolumn{2}{|c|}{ Gene Xpert technique } & \multirow[b]{2}{*}{ Total } & \multirow[b]{2}{*}{$\begin{array}{l}\text { Карра (k) } \\
(95 \% \text { CI) }\end{array}$} & \multirow[b]{2}{*}{ P-value } \\
\hline & & Positive & Negative & & & \\
\hline Positive & & 17 & 27 & 44 & & \\
\hline Negative & & 0 & 114 & 114 & $0.48(0.32-0.63)$ & $<0.001$ \\
\hline Total & & & 141 & 158 & & \\
\hline
\end{tabular}

\section{DISCUSSION}

Tuberculosis in urine was found to be high in this study suggesting a major challenge in the diagnosis of TB in patients who are TB/HIV coinfected. This may be associated with poor diagnosis and delayed treatment. These findings are in agreement with other studies recently reported from South Africa, which found high TB prevalence using urine from immunosuppressed persons and recommended a further investigation of creatinine ${ }^{13}$. The hemoglobin level (12.0) was within the normal ranges implying that participants were not anemic.

This study established that clinical symptoms; weight loss, TB, Pneumonia, URTI and those with a combination of TB or Pneumonia or Meningitis diagnosed. Also infiltrate, milliary $\mathrm{TB}$ and opacities. This is because the clinical presentation of MTB in AIDS can resemble that of non-AIDS patients and MTB can often be the first AIDSdefining illness, particularly in regions where the incidence of MTB is high in the general population. Chest $\mathrm{x}$-ray (CXR) is mostly used as the diagnostic tool for TB but HIV/TB co-infected patients have normal CXR results despite the fact that their culture results are positive $14,15$. Tuberculosis should be suspected in patients with fever, cough, night sweats and weight loss, regardless of chest roentgenogram findings ${ }^{16,17}$.

Gene Xpert test, patients were identified as TB positive of which $94 \%$ were MTB detected low and $6 \%$ were MTB detected medium. The study has proven that urine Xpert is a useful tool in detecting TB/HIV patients living in western Kenya and offer incremental yield when it comes to diagnosis of tuberculosis using urine. Rifampicin resistance was not detected and all patients were sensitive to drugs. This designates no drug resistance and the diagnosed cases are treatable.

This current study showed that patients tested positive for TB using LAM. LAM in urine indicates the presence of disseminated tuberculosis. i.e. tuberculosis other than pulmonary tuberculosis, mostly affecting the kidney. The organ distribution of Mycobacteria tuberculosis (MTB) in AIDS is widespread.

It was also noted that presence of protein (proteinuria) or blood (hematuria) in urine was significantly associated with TB positivity based on LAM test.HIV patients who have severe immunosuppression have high bacillary load leading to shedding of LAM in urine and also due to HIV associated nephropathy (HIVAN), a 
nephrotic syndrome signaling renal abnormalities and a failure in glomerular filtration mechanism. HIVAN is characterized by marked proteinuria and a rapid progression to renal failure and end stage renal disease (ERSD) ${ }^{18}$.Immune complex mediated glomerulonephritis (proliferative glomerulonephritis) may also occur in HIVinfected patients presenting with proteinuria and renal failure ${ }^{19}$.

\section{CONCLUSSION AND RECOMMENDATION}

In conclusion, our study shows that urine Xpert MTB/RIF® is a highly sensitive and specific tool for diagnosis of TB using urine in severely immunosuppressed individual Western Kenya population where sputum diagnosis is not possible. On the other hand urinary LAM strip test is highly sensitive in diagnosis of tuberculosis in HIV patients particularly in immunosuppressed individual.

\section{ACKNOWLEDGEMENTS}

The authors of this paper acknowledges the contribution of Maseno University and MTRH.

\section{REFERENCES}

1. WHO. TB/HIV facts 2012-2013. Accessed $09^{\text {th }}$ June 2015. Available at www.who.int/hiv/topics/tbhiv_facts_2013/ en/

2. WHO. (2011)Rapid Implementation of the XpertMTB/RIF Diagnostic Test. Publication number WHO/HTM/TB/2011.2.2. World Health Organization, Geneva, Switzerland.

3. Moore D. L. C., Ekwaru P, Were W, Mwima G. and Solberg P. (2007) Prevalence, incidence and mortality associated with tuberculosis in HIVinfected patients initiating antiretroviral therapy in rural Uganda. AIDS21,713-719.

4. Boehme C.C., Hillemann D, Nicol M.P., ShenaiS, and (2010)Krapp F. Rapid Molecular Detection of Tuberculosis and Rifampin Resistance. $N$ Engl J. Med363,1005-1015.
5. Dawson R, M. P., Edwards D.J., Bateman E.D., Bekker L.G., and Wood R. (2010) Chest radiograph reading and recording system: evaluation for tuberculosis screening in patients with advanced HIV. Int. J.Tuberc Lung Dis14(14),52-18.

6. Lawn S.D., Vogt M.and Wood R. (2012) Diagnostic accuracy of a low-cost, urine antigen, point-of-care screening assay for HIV-associated pulmonary tuberculosis before antiretroviral therapy: a descriptive study. Lancet Infect Dis 12, 201-209.

7. Monkongdee P. M. K., Cain K.P., Tasaneeyapan T, Nguyen H.D. and Nguyen T.N. (2009) Yield of acid fast smear and mycobacterial culture for tuberculosis diagnosis in people with human immunodeficiency virus. Am J RespirCrit Care Med180,903-908.

8. Reid M.J. S. N. (2009). Approaches to tuberculosis screening and diagnosis in people with HIV in resource-limited settings. Lancet Infect Dis9, 173-184.

9. Green C. H. J., Talbot E, Mwaba P, Reither K. and Zumla A.I. (2009) Rapid diagnosis of tuberculosis through the detection of mycobacterial DNA in urine by nucleic acid amplification methods. Lancet Infect Dis 9,505-511.

10. Hillemann D, R.-G. S., Boehme C. and Richter E. (2011) Rapid Molecular Detection of Extrapulmonary Tuberculosis by the Automated GeneXpert MTB/RIF System. J. Clin. Microbiol49,1202-1205.

11. Boehme C.C., Nabeta P, Michael J.S., and Gotuzzo E. (2011) Feasibility, diagnostic accuracy, and effectiveness of decentralised use of the Xpert MTB/RIF test for diagnosis of tuberculosis and multidrug resistance: a multicentre implementation study. Lancet377, 14951505.

12. Lawn S.D., Vogt M, Ghebrekristos Y, Whitelaw A. and Wood R. (2012) Characteristics and early outcomes of 
patients with Xpert MTB/RIF-negative pulmonary tuberculosis diagnosed during screening before antiretroviral therapy. Clin Infect Dis54(8), 1071-1079.

13. Peter J.G., van Zyl-Smit R, Haripersad A. andMottay L. (2012) Diagnostic accuracy of a urine LAM strip-test for TB detection in HIV-infected hospitalised patients. The European Resp. J. 40(5),1211 1220.

14. Wilson, D., Nachega, J,Morroni, C, Chaisson, R. and Maartens, G. (2006) Diagnosing smearnegative tuberculosis using case definitions and treatment response in $\mathrm{HIV}$-infected adults. Int $J$ Tuberc Lung Dis10(1), 31-38.

15. van Cleeff, M.R., Kivihya-Ndugga,L.E., Meme, H. Odhiambo, J.A.andKlatser, P.R. (2005) The role and performance of chest $\mathrm{X}$-ray for the diagnosis of tuberculosis: a cost effectiveness analysis in Nairobi, Kenya. BMC Infect Dis 5,111.

16. Klatt E.C., Nichols L. and Noguchi T.T. (1994) Evolving trends revealed by autopsies of patients with AIDS. Arch Pathol Lab Med 118, 884-890.

17. Havlir D.V. Barnes P.F. (1999) Tuberculosis in patients with human immunodeficiency virus infection. $N$ Engl J Med 340,367-373.

18. Herman E.S. and Klotman P.E. (2003) HIV-associated nephropathy: epidemiology, pathogenesis, and treatment. SeminNephrol23, 200-2008.

19. Kimmel P.L., Phillips T.M., FerreiraCenteno A, Farkas-Szallasi T, Abraham A.A. and Garrett C.T. (1993) HIVassociated immune-mediated renal disease.

Kidney Int44, 1327-1340. 